\title{
Hand-Exoskeleton Assisted Progressive Neurorehabilitation using Impedance Adaptation based Challenge Level Adjustment Method
}

\author{
Anirban Chowdhury, Student Member, IEEE, Shyam Sunder Nishad, Yogesh Kumar Meena, Member, IEEE, \\ Ashish Dutta, Member, IEEE, and Girijesh Prasad, Senior Member, IEEE
}

\begin{abstract}
This paper presents an underactuated design of a robotic hand exoskeleton, and a challenge based neurorehabilitation strategy. The exoskeleton is designed to reproduce natural human fingertip paths during extension and grasping, keeping minimal kinematic complexity. It facilitates an impedance adaptation based trigged assistance control strategy by switching between active non-assist and passive assistance modes. In the active non-assist mode, the exoskeleton motion follows the applied fingertip forces based on an impedance model. If the applied fingertip forces are inadequate, the passive assistance mode is triggered. The impedance parameters are updated at regular intervals based on the user performance, to implement a challenge based rehabilitation strategy. A six-week long hand therapy, conducted on four chronic stroke patients, resulted in significant $(p$-value $<0.05)$ increase in force generation capacity and decrease $(p$-value $<0.05)$ in the required assistance. Also, there was a significant $(p$-value $<0.05)$ increase in the system impedance parameters which adequately challenged the patients. The change in the Action-Research-Arm-Test (ARAT) scores from baseline was also found to be significant $(p$-value $<0.05)$ and beyond the minimal clinically important difference (MCID) limit. Thus the results prove that the proposed control strategy with has the potential to be a clinically effective solution for personalized rehabilitation of poststroke hand functionality.
\end{abstract}

Index Terms-impedance adaptation, trigged assistance, Neurorehabilitation, Hand-Exoskeleton, Stroke.

\section{IntRODUCTION}

Motor impairment of the upper limb, in terms of loss of ability to use the fingers due to stroke, is a major cause of disability worldwide. For effective motor recovery, the stroke survivors need to undetake intensive physiotherapy using their paretic limbs, involving active engagement [1]. In most cases, effective recovery is delayed and sometimes hindered, as the conventional therapies are not adequately intense and engaging for the patients, or the trained professionals are unavailable [2].

In recent years, robotic therapies have been shown to provide intense repetitive exercise for the patients with partial or no involvement of a human therapist [3]. These are easily deployable and can also provide very reliable quantification of the rehabilitation progress [4]. Although the robotic rehabilitation for upper-limb poststroke impairment is often recommended

A. Chowdhury, A. Dutta and S. S. Nishad are with the Department of Mechanical Engineering, Indian Institute of Technology Kanpur, Kanpur208016, India, e-mail: anir@iitk.ac.in

Y. K. Meena is with Department of Computer Science, Swansea University, Swansea, SA2 8PP, U.K.

G. Prasad is with School of Computing Engineering and Intelligent Systems, Ulster University, Derry Londonderry, BT48 7JL, U.K. by major health care organizations such as American Heart Association, the personalization of the therapeutic process according to the specific needs of a patient is a major problem [5]. In our study we have focused on the rehabilitation of the distal part of the upper-limb, particularly the finger flexion/extension. Based on the structure and the actuation systems the exoskeletons are found mainly in two categories: a major portion of them are cable actuated [6], [7], [8], [9], [10], [11], and a minority of them are driven by rigid structures or linkages [12], [13], [14], [15]. Recently pneumatic actuators [16] are also being used to build soft exoskeletons. Other than the exoskeletons haptic devices such as ReHapticKnob [17] are also in use for hand rehabilitation. The exoskeletons also vary in their design complexity, as some of them go for the full phalanges [15] such as HANDEXOS [18], ExoK'ab [14] and BiomHED [9], while others such as CAFE [19], SNU Exo-Glove deal with a subset of it [7], [10]. Sometimes, a sub-set for full phalanges is also driven in couple [16] in order to reduce the complexity, such as GENTLE/G [13] and HandSOME [20]. The individual finger modules are also actuated in different ways as per the need of the therapeutic exercise. Hand exoskeletons such as [6], [14], [15] actuates all the joints of a finger module, while the finger modules of [8], [7], [16] are underactuated. The compromise between making actuating all the fingers and actually all the joints of a finger module has to be made, as incorporating both them (e.g. [15]) makes the exoskeleton bulky, and limits the portability and wearability of it. The actuations systems have also their individual limitations. In the cable driven systems the tension in the wire doesn't always correctly reflect the summation of the joint torques and also the tension in the wire often gets disturbed by the arm and wrist movements [11]. The rigid linkages although suitable for exerting higher forces for challenging rehabilitation exercises, often constrains the non-actuated joints and disrupt the natural motion, thereby making system uncomfortable for the patients [16]. A detailed review of the existing wearable haptic systems for the fingertip and hand is given in [21]. Although several robotic hand exoskeletons already exist, design of light-weight, fully wearable, low-cost and safe hand exoskeletons facilitating natural finger motion, and the development of engaging and challenging training paradigms for neurorehabilitation still require extensive research [1].

Apart from diverse design strategies there are also a variety of control approaches for hand exoskeletons. For over a decade 
the mechanical impedance models are being used for assistas-needed control of the exoskeletons. Its central objective is to encourage the voluntary participation of the user in following a desired trajectory, as the controller intervenes by producing an assistance force which increases only when the user deviates from the desired trajectory, and doesn't intervene otherwise [22]. Thus it avoids the slacking effect, which hinders the process of motor skill learning [23]. One of the main challenges in assist-as-needed approach is to find the ideal joint angle trajectory for the required limb movement, as it forces the user to follow a desired trajectory [22]. This also reduces free interaction between the human and the device, rather than promoting it, which is beneficial for an effective motor recovery [24]. Moreover, to figure out the minimal assistance level in real time, the assist-as-needed controllers need to solve optimization problems which increase the computational and hardware complexity compromising the agility of the system design.

Another control approach involving a triggered assistance is considered as a variant of the assist-as-needed principle [22]. In this approach the assistance is triggered when one or more performance criteria hits a predefined threshold, while the participants initially attempt the movement without robotic intervention. The performance criteria are usually set as muscle activity, limb-velocity, tracking error, elapsed time, and force generation or a combination of these [25]. The exoskeletons such as HWARD and RUPERT used the threshold as elapsed time [26], [27]. In the force triggered approaches the robotic assistance is provided when the participants are able to generate a force larger than a threshold force [28], [29]. In contrast, some researchers followed the reverse approach, which is to initiate the robotic assistance if the applied torque by the participant is under a threshold torque for a certain period of time [30], [31]. Although both the forward and reverse approaches of triggered assistance are intended for encouraging the active participation of the users, the forward approach (i.e. triggering the assistance when the participant meets the performance criteria) is intuitively less rewarding as the assistance is triggered when the performance is higher i.e. the requirement of assistance is less. However, both forward and the reverse approach of triggered assistance have the tendency to make the participants apparently passive extending the assistance phase. Although many exoskeletons described here provides active/passive assistance to the patients, the haptics aspect of the system is often neglected. There are a few devices such as ReHapticKnob [17], wherein the output impedance is modulated autonomously in order to vary the assistance/resistance level of the system and thereby adjusting the challenge level of the therapeutic task without the intervention from a human therapist.

In the light of the above mentioned structural and control system designs, the paper proposes a hand-exoskeleton for stroke rehabilitation which is agile in both the structural and control aspects and validates its potential clinical effectiveness through pilot clinical trial on stroke patients. The developed hand exoskeleton is a four-bar linkage driven underactuated system capable of driving the index and middle fingers in a coupled fashion and thumb independently. It follows a triggered assistance approach (named here as impedance adaptation based trigged assistance) in order to make the design of the control strategy agile enough by reducing the computational and hardware complexity involved in conventional assist-as-needed approach. Additionally, The triggered assistance strategy reacts interactively to the volitional effort of the patients, without constraining them to follow a desired trajectory (as in the case of the conventional implementation of assist-as-needed approach) or force pattern, to incorporate the idea of free interaction, which is often considered advantageous in a rehabilitation scenario [24]. The rehabilitation approach, proposed in this paper is a challenge based one, in which, the challenge level is modified by varying the virtual impedance of the exoskeleton system based on the force generation capacity of the user. The advantage of this strategy is that it maximizes the patient's engagement as it optimally sets the difficulty level so that the patients do not get frustrated by a too easy or a too difficult task [32]. The novel contributions of the study are as follows:

1) A novel challenge based neurorehabilitation strategy, by adapting the mass and the damping parameters of the impedance model, to facilitate to the stroke patients, a performance based progressively challenging physical exercise;

2) a fusion between the active non-assist and passive assistance mode based control strategy for promoting free interaction and

3) a new performance metric based on force generation capacity of the patients, which also correlated with the motor recovery measure for grip-strength.

The rest of the paper is organized as follows. In Section II, the design and development of the hand exoskeleton is discussed. The impedance adaptation based trigged assistance control strategy and its experimentation on stroke patients are discussed in Section III. The impact of the proposed control strategy using hand exoskeleton on stroke patients is presented in Section IV, while the discussion and conclusion based on the results obtained, are stated in Sections $\mathrm{V}$ and VI respectively.

\section{Exoskeleton Design}

Human fingertip path during grasping and extension tasks is non-circular [34]. The simplest mechanism which generates this path is a planar four-bar linkage. To accomplish this design, (i) human finger motion of healthy subjects was recorded (Figs. 1,2) while they performed full extension and grasping, and (ii) four-bar linkages for the thumb and index finger were synthesized optimally for path generation so as to match the recorded fingertip trajectories (Fig. 3). The input link $\mathrm{O}_{2} \mathrm{~A}$ is attached to the servomotor's shaft for actuating the exoskeleton. The method employed by Nishad et al. [33] for three DOF per finger exoskeleton module design is adapted for this single DOF finger exoskeleton. The optimal link lengths are given in Table II.

A CAD model of the exoskeleton was developed and then a prototype was fabricated using selective laser sintering. 


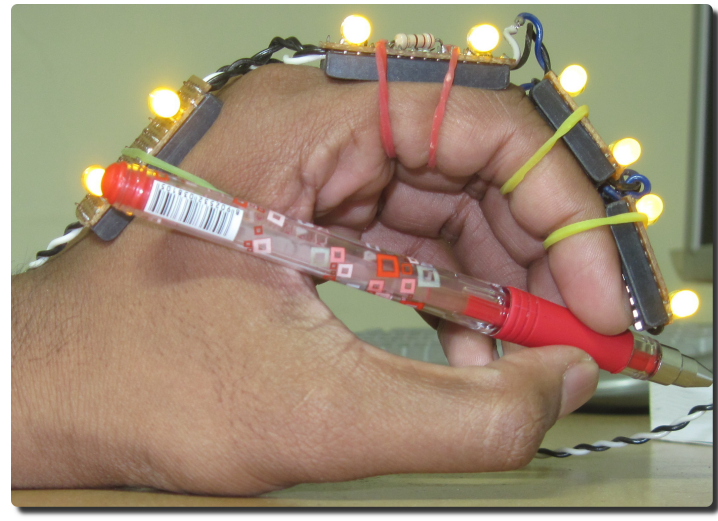

Fig. 1. Finger motion capture setup [33]: Eight LED markers. Motion is recorded using a single camera.

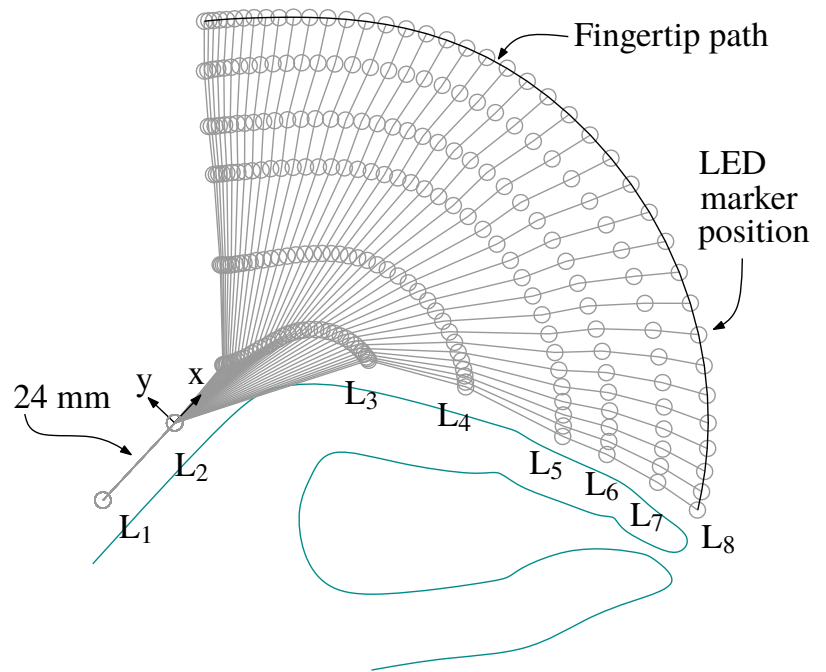

Fig. 2. Human index fingertip path for extension/grasp motion: The oval markers represent the positions of LED markers $\mathrm{L}_{1}-\mathrm{L}_{8}$ placed on a finger for data recording. The fingertip curve traced is non-circular. The first two markers (leftmost) are on the back of the hand. The data points are transformed with respect to the coordinate system x-y formed by these two markers. 24 such data-sets were collected from healthy subjects for the design computations.

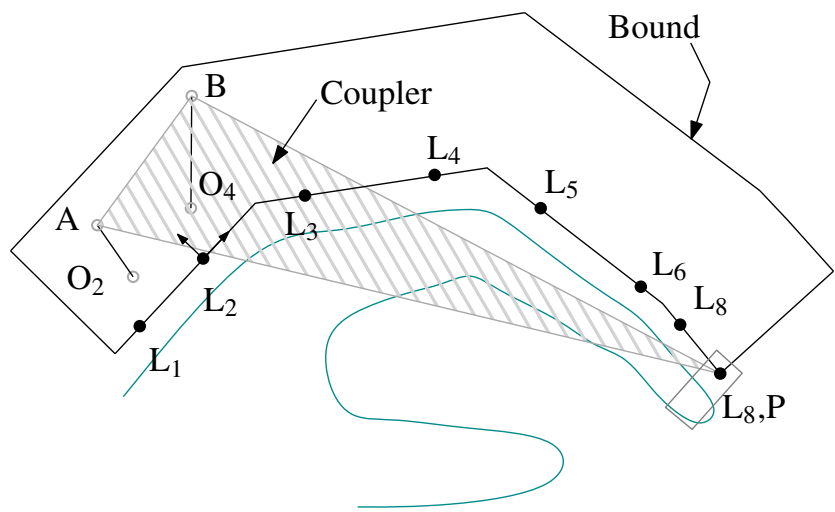

Fig. 3. Schematic Sketch of the finger exoskeleton design using a four-bar linkage: The four-bar linkage is optimized to generate the path of the LED $\mathrm{L}_{8}$, and is constrained to lie as close to the finger as possible, by introducing a 'Bound' constraint.

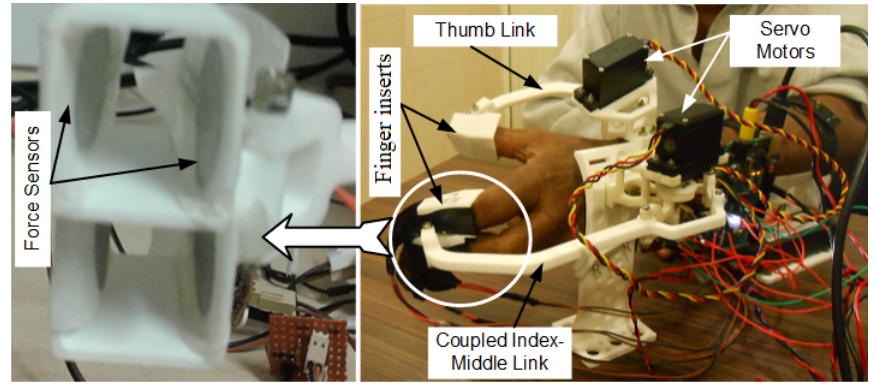

Fig. 4. View of the hand exoskeleton worn on the hand of a person. The index and middle fingers are inserted into the finger-insert of the coupled indexmiddle link of the exoskeleton and the thumb is inserted into the finger-insert of the thumb link. The inside of the finger insert is zoomed at the left side with an arrow to show the force-sensor attachments, where the finger-tip of the user is actually touching. The servo motors as depicted in the figure are driving the each individual link. When the user tries to grasp or extend the fingers the applied forces are picked up by the force sensors placed in either direction and then the exoskeleton controller converts them into appropriate motion.

TABLE I

VALUES OF PARAMETERS FOR GA() FUNCTION IN MATLAB

\begin{tabular}{ll}
\hline \hline Parameters & Values \\
\hline Population Size & 120 \\
Cross-Over Fraction & 0.95 \\
NonlinConAlgorithm & penalty \\
Mutation Function & mutationadaptfeasible \\
Generations & 2000 \\
Stall Generations & 2000 \\
Function Tolerance & $10^{-6}$ \\
Constraint Tolerance & $10^{-6}$ \\
\hline
\end{tabular}

The index and middle fingers are coupled and actuated by a single four-bar linkage and the thumb by another fourbar linkage. This was done, as it was observed that, the index and middle fingertip paths are similar and they move on the same plane. Hence, this is an underactuated design with two active DOF, one for the index and middle fingers combined, and one for the thumb. Two fingertip inserts are attached through passive revolute joints to the exoskeleton finger modules, inside which the fingers are inserted during exercise. The fingertip inserts allow for some variation of finger lengths and hence the same exoskeleton can be used by different persons having slightly different finger lengths. The fixture (Fig. 4) for the finger exoskeletons includes an arm-rest support for providing comfort during rehabilitation exercises.

Exoskeleton designs for rehabilitation are often not focused

TABLE II

Optimization Results

\begin{tabular}{lrr}
\hline \hline Link Lengths (mm) & Index & Thumb \\
\hline $\mathrm{O}_{2} \mathrm{O}_{4}$ & 40.3 & 14.7 \\
$\mathrm{O}_{2} \mathrm{~A}$ & 32.8 & 26.3 \\
$\mathrm{O}_{4} \mathrm{~B}$ & 35.3 & 25.4 \\
$\mathrm{AB}$ & 51.0 & 20.8 \\
$\mathrm{AP}$ & 135.2 & 107.0 \\
$\mathrm{BP}$ & 184.3 & 127.5 \\
\hline \hline
\end{tabular}


TABLE III

Design FeAtures

\begin{tabular}{ll}
\hline \hline Features & Details \\
\hline $\begin{array}{l}\text { Form Factor } \\
\text { Weight }\end{array}$ & $\begin{array}{l}\text { Wearable and portable; follows body shape } \\
\text { Purpose }\end{array}$ \\
$\begin{array}{l}\text { Constrains fingers to follow natural fingertip path } \\
\text { in extension and grasping motion, without putting } \\
\text { constrains on the timings, to promote free interaction } \\
\text { between the user and the device }\end{array}$ \\
$\begin{array}{l}\text { Easy to wear and tolerable for long time } \\
\text { End-effector } \\
\text { Actuation }\end{array}$ & $\begin{array}{l}\text { Rigid slots for accommodating fingertips } \\
\text { Servomotor with four-bar mechanism }\end{array}$ \\
\hline \hline
\end{tabular}

on wearability [35], [36], while in our case it is considered one of the key design aspects, so that its application can also be extended to daily living activities. Pacchierotti et al. [21] defined the wearable exoskeletons on the basis of their form factor, weight, shape, area of interest and ergonomics. A desirable characteristic for the form factor of an exoskeleton is that it should not hinder the natural movements and should follow the shape of the body part on to which it is worn. The exoskeleton, designed in this study acts only on the finger pads and follows the natural human finger trajectory using the four-bar mechanism. Hence, no obstruction occurs during the natural motion of the fingers. The unique design of the armrest with the two stands makes it easy and comfortable to wear for long time making it fully wearable and portable (see Fig. 4). The arm-rest follows the natural shape of the forearm. 'Velcro straps' keep the exoskeleton attached to the hand and the forearm. A patient with hemiplegia using the exoskeleton is shown in Fig. 5.

The device weighs $410 \mathrm{~g}$. Two Hitec HS5685MH servo motors with $180^{\circ}$ rotation are used to actuate the exoskeleton. These motors weighing $60 \mathrm{~g}$, are small in size $(41 \times 20 \times 38$, in $\mathrm{mm}$ ) and are capable of exerting $12.9 \mathrm{~kg} \mathrm{~cm}$ torque at $7.4 \mathrm{~V}$. The device is also back-drivable, a feature which was required during the experiments to calculate the initial impedance parameters. Among the lightweight exoskeletons, the device built by Arata et al. [37] weighs $320 \mathrm{~g}$, which uses compliant mechanisms, while the soft robotic glove using hydraulic actuators designed by Polygerinos et al. [38] weighs $285 \mathrm{~g}$.

A patient with hemiplegia using the exoskeleton is shown in Fig. 5. There are several problems which could arise while applying the device to a paretic hand such as: 1) as the upperlimb of the patients is generally weak, aligning the device with the orientation of the hand is a little cumbersome, 2) due to the weakness in the hand and finger muscles, the fingers can slip out of the finger-inserts if not properly pushed inside or the wrist and arm are loosely fastened with the exoskeleton, 3 ) as the exoskeleton is for prolonged use, any part of it should not be pinching or putting pressure on the hand making any sort of discomfort while doing the excercise. The design of the arm-rest is very useful here, and unique in the sense that most of the existing designs of the hand exoskeleton do not have such an arm rest which covers from the back of the hand to the mid-lower arm with two stands which hold them fix on a table. Generally, the impaired upper limb is weak in a patient and

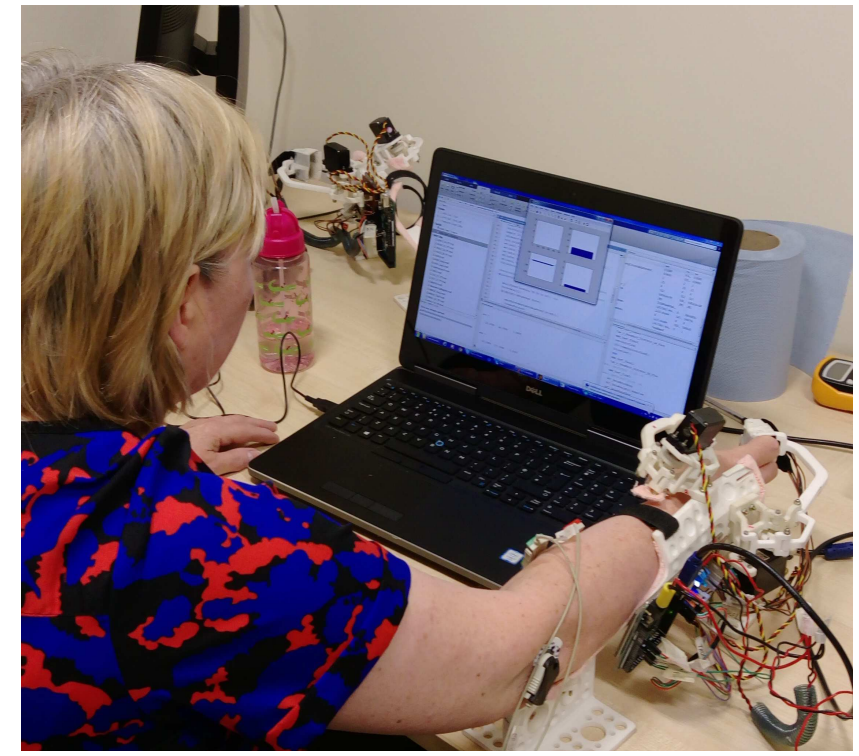

Fig. 5. Experimental setup: A participant is doing exercise, wearing the hand exoskeleton on her impaired hand. The applied fingertip force is displayed on the screen.

it needs such a support system which not only will keep the hand steady and comfortable while doing the therapeutic task, but could also be easily detached during activities of daily living (ADL). A fixed support system generally will restrict the portability of the exoskeleton.

A questionnaire to evaluate the usability of the handexoskeleton was also given to the participants, to get the qualitative feedback of their experience of using it. The responses show that the exoskeleton was easy to put on with minimal external assistance requirement. The exoskeleton was also comfortable to wear for long duration, and there was no discomfort in performing the finger extension and grasping motions while using the exoskeleton.

The design features are summarized in the table III.

\section{IMPEDANCE ADAPTATION BASED TRIGGED ASSISTANCE}

\section{A. Control System Overview}

Two force sensors (FSRs), mounted on the upper and lower inner walls of each finger-insert, are used to sense the force exerted by the participant in the directions of grasping and extension. From the readings of the four force sensors (two for index finger and two for middle finger), the net force acting in a particular direction (grasping or extension) is calculated using (1).

$$
F_{\text {net }}=\left(F_{\mathrm{mp}}+F_{\mathrm{ip}}\right)-\left(F_{\mathrm{ma}}+F_{\mathrm{ia}}\right)
$$

Here, $F_{\mathrm{ip}}, F_{\mathrm{mp}}$ are the force readings on the posterior side of the index and middle fingertips respectively and $F_{\mathrm{ia}}, F_{\mathrm{ma}}$ are the force readings for the anterior side. Thus, if the net force $F_{\text {net }}>0$, then it is considered to be acting in the extension direction and if $F_{\text {net }}<0$ then the net force is considered to be acting in the grasping direction. This net force is compared with a threshold force level $F_{\text {th }}$. To set the threshold force we asked the participants to keep their hand passive and moved the exoskeleton for a finger open and close motion. Then we 
took the average of all the forces which was passively reflected on the force sensor to calculate the threshold force level. Thus the value of $F_{\text {th }}$ turned out to be $0.02 \mathrm{~N}$. As the participants were asked not to make a deliberate attempt of movement during this experiment, the $F_{\text {th }}$ can be safely assumed to be the force reflected by slight vibration or the passive resistance offered by the fingers. In this regard it is to be noted that all the participants had negligible spasticity in their fingers. The threshold force level ensures that the applied force is not due to any random vibration in the fingers, which doesn't represent patient's volitional effort. The user would be able to drive the exoskeleton deliberately only when, the net fingertip force $F_{\text {net }}$ applied by him/her exceeds the threshold force $F_{\text {th }}$, i.e.,

$$
\left|F_{\text {net }}\right|>\left|F_{\text {th }}\right|
$$

In this case, the exoskeleton is said to operate in active nonassist mode, in which an impedance model (Section III-B) is used to compute the exoskeleton motion from the fingertip force. In case the inequality (2) is not satisfied at any point of time, the controller starts a watchdog $\left(w_{\mathrm{t}}\right)$ and waits for a particular period of time $w_{\text {th }}$ before issuing the flag that the user failed to generate the required force to drive the exoskeleton. The $w_{\text {th }}$ was empirically set to $500 \mathrm{~ms}$ to give the participant enough time to respond. In this case, the exoskeleton is said to operate in passive assistance mode, in which, the user gets full assistance from the exoskeleton controller, as the fingers are moved in the direction of motion with a default velocity $\left(\dot{\theta}_{\text {def }}\right)$ to complete the ongoing grasping/extension. The default velocity $\left(\dot{\theta}_{\text {def }}\right)$ was set to $9.27 \mathrm{deg} / \mathrm{s}$ for the crank, to give the user sufficient time for proprioception. During the passive assistance the exoskeleton was driven in velocity control mode, where it maintained a fixed velocity of $9.27 \mathrm{deg} / \mathrm{s}$ until it receives further input from the user (i.e. until $F_{n e t}>F_{t h}$ ). At any point of time during the passive assistance mode, if the user is able to apply enough fingertip force in the direction of motion to drive the exoskeleton (i.e. satisfying (2)), the controller switches to the active non-assist mode. The fact that the exoskeleton controller provides assistance only when, the user fails to drive the exoskeleton deliberately, it facilitates the impedance adaptation based trigged assistance policy, an essential requirement for motor skill learning.

\section{B. Impedance Model}

Impedance models are widely used to convert the applied force to control the position of robots [39] in a robot human cooperation task. Perez-Ibarra et. al. [40] proposed an ankle rehabilitation strategy based on an adaptive impedance control model. They used damping and stiffness parameters only, and updated the stiffness parameter based on the patient's performance in a video game. Metzger et al. [1] reported an assessment driven selection and adaptation of the exercise difficulty in a robot assisted therapy. They also used an impedance model that had only a stiffness parameter which was varied to evaluate a patient's ability to perceive and differentiate haptic stimuli during active object grasping. As per the literature in [41] and [42], including a spring in the impedance model can impede the human-robot interaction.
This is because the spring essentially absorbs energy and gives it back to the system, thus, causing oscillations. Hence, the impedance model considered here consists of the inertia and damping terms only, to provide the best cooperation between the robot exoskeleton and a human. Each finger module of the exoskeleton has only one active degree of freedom, the input link angle $\theta$ of the four-bar mechanism. This $\theta$ is related to the net force $F_{\text {net }}$, to form the proposed impedance model as,

$$
F_{\text {net }}=M \ddot{\theta}+C \dot{\theta}
$$

The estimates of the patient's $M, C$ parameters were calculated with reference to a healthy individual's $M, C$ parameters. In order to estimate the $M, C$ parameters specific to a healthy individual, the following experiments were conducted. A group of 10 healthy people between the age group of $20-50$ years were asked to perform the grasping and extension wearing the exoskeleton while its actuators were inactive. As the servomotors used are back-drivable, they can be moved even in the power-off condition. The fingertip force and the corresponding change in the actuator angle were recorded. The variation of the fingertip force $\left(F_{\mathrm{H}}\right)$ with the actuator angle, averaged over all participants is shown in Fig. 6a. An oneway ANOVA test between the force profiles of all the healthy participants resulted in $p$-value $>0.05$, meaning that there is no significant difference between the means of these force profiles. Therefore, we took the average of all the force profiles to set the $F_{H}$. From the actuator angular position, the velocity and the acceleration were computed numerically (Fig. 6b). The actuator velocity, acceleration and fingertip force data were then used to estimate the $M, C$ parameters of the impedance model (3), by solving a non-linear curve fitting problem using a non-linear least square solver. The problem was formulated as follows:

$$
\min _{M, C} \sum_{i=1}^{N}\left(M \ddot{\theta}_{i}+C \dot{\theta}_{i}-F_{\mathrm{H}_{i}}\right)^{2}
$$

where $N$ is the total number of data points. The sampling rate of the data acquisition device was $50 \mathrm{~Hz}$, which gave a total of $N=104$ data points for one grasping motion taking $2.08 \mathrm{~s}$. The algorithm to solve the non-linear least square problem is the subspace trust region method based on the interior-reflective Newton method. Here, we used the MATLAB implementation of the solver i.e. lsqcurvefit(). The average $M, C$ parameters for a healthy individual interacting with the exoskeleton were found to be $5.3 \mathrm{Ns}^{2} / \mathrm{deg}$ and $0.82 \mathrm{Ns} / \mathrm{deg}$. Using these values, we can numerically integrate Eq. (3) using the second order Runge-Kutta method, to get the current position and velocity of the crank, given the previous state of the position, velocity of the crank and the applied force. Thus, the net force $F_{\text {net }}$ is converted into the exoskeleton motion using the impedance model. Figure $6 \mathrm{c}$ shows the reconstructed force profile after the estimation of $M, C$ of the healthy participants, which is plotted over the original force profile to check the validity of the $M, C$ estimates.

\section{Clinical Intervention Protocol}

In this study, we have conducted a single-arm clinical trial comprising of 4 hemiparetic stroke patients who underwent 


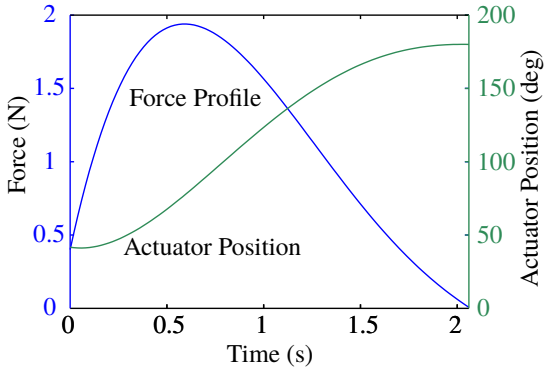

(a)

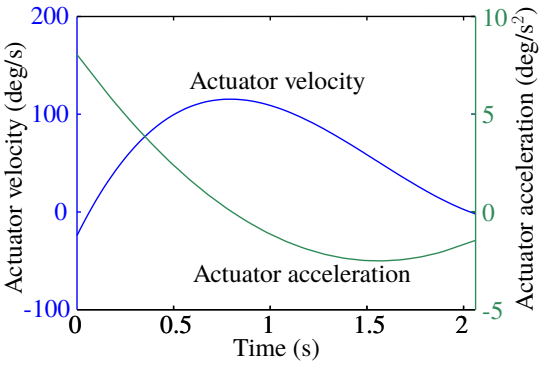

(b)

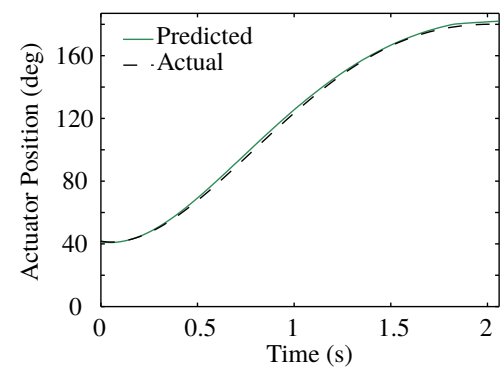

(c)

Fig. 6. Different kinematic and dynamic characteristics of a healthy individual during grasping motion wearing the exoskeleton: (a) fingertip force and actuator position, (b) actuator velocity and acceleration. An impedance model for the grasping motion wearing the exoskeleton was developed using these characteristics. (c) The reconstruction of the actuator position profile during grasp. Black dotted line is the experimental position profile and green solid line is computed using the impedance model.

the same intervention for 6 . The intervention was given three times per week and consists of two stages. The first stage was the Physical Practice (PP) stage of 30 min followed by a brain-computer interface (BCI) supported mental Practice (MP) stage of almost an hour including the BCI calibration time of around $16 \mathrm{~min}$. Participants were made to sit on a chair in an upright position at about $0.5 \mathrm{~m}$ distance from the computer screen with the exoskeleton worn on their impaired hand (Fig. 5).

In the PP stage the participants were instructed to execute extension or grasping motion when the corresponding cue signal was provided on the screen. Depending on the fingertip force exerted by the patient, the exoskeleton would operate either in active non-assist mode or passive assistance mode. We have termed one such grasping followed by an extension as a practice unit (PU). This grasping and extension cycle was repeated one after another for $30 \mathrm{~min}$. Participants also got real-time feedback of their applied fingertip force on the computer screen so that they could take corrective measures to drive the exoskeleton more efficiently. The average of all the $F_{n e t}$ (where, $F_{n e t}>F_{t h}$ ) is considered as $P U_{\text {score }}$, i.e. the average net force (greater than threshold force) generated in a PU. Now a PP session of $30 \mathrm{~min}$ is composed of many such PUs (the number of PUs depends on the performance of the participant during that PP session). Thus the session score $(S S)$ is defined as the average of all the $P U_{\text {score }}$ in 5.

$$
S S=\frac{\sum_{n=1}^{N_{p u}} P U_{\text {score }}(n)}{N_{p u}}
$$

where, $P U_{\text {score }}(n)$ is the $P U_{\text {score }}$ of $n_{t h} \mathrm{PU}$ during a $\mathrm{PP}$ session. $N_{p u}$ is the total number of PUs in a PP session. Before starting a new session, the participants were given the target to achieve better score than in the previous session. To make the rehabilitation paradigm more challenging, a performance based impedance update strategy was implemented in the exoskeleton controller. The performance was measured using the session score $(S S)$, based on which, the impedance parameters for the next session were updated (Section III-D). This policy continuously challenges the participant by increasing the difficulty level of the task as the performance level is enhanced.
In the MP stage the participants were given a hybrid-BCI based multimodal neurofeedback contingent to the simultaneous activations in the EEG and EMG signal measured by a spectral power correlation between the two. In one particular session of the MP, there were five runs of approximately $7 \mathrm{~min}$ $30 \mathrm{sec}$ each consisting of 40 trials. Each trial starts with a three second rest period, followed by the presentation of the cue in each of which the participants were asked to perform a left/ right-hand grasp attempt. Although the cue remains for two seconds, the participants were asked to perform the task until five seconds after the presentation of the cue. Among the five runs, first two runs were for calibrating the $\mathrm{BCI}$ system and the subsequent three runs were for giving online neurofeedback based on the EEG-EMG pattern classifier trained during the calibration stage. For the online neurofeedback runs, visual and proprioceptive feedbacks were provided through the screen and hand-exoskeleton, respectively, during the last three seconds of the task period. Details on the methods used during the BCI based mental practice can be found in authors' previous works [43], [44]. The current study focuses only on the design of the exoskeleton and its impedance adaptation based trigged assistance control, to provide a challenge based rehabilitation strategy, implemented using impedance parameter adaptation. All results in this paper relate to the physical practice part only.

\section{Impedance Parameters Updating Method}

This method adapts the impedance parameters so that the patients' lower force generation capacity can be augmented by considering the performance of a healthy human being as a reference. In this method, the average force profile $F_{\mathrm{H}}$ of a healthy person is scaled so that, the force profile $F_{\mathrm{S}}$ required from the patient in the next session has its mean equal to the patient's previous session score $S S$. Thus:

$$
F_{\mathrm{s} i}=\frac{F_{\mathrm{H} i}}{\operatorname{mean}\left(F_{\mathrm{H}}\right)} S S \quad i=1,2, \ldots, N
$$

The new impedance parameters $M$ and $C$ are computed using the same procedure as discussed in section III-B. The trajectory data $\dot{\theta}$ and $\ddot{\theta}$ are of the healthy person as stated above, while the force profile $F_{\mathrm{H}}$ is replaced by the new profile 


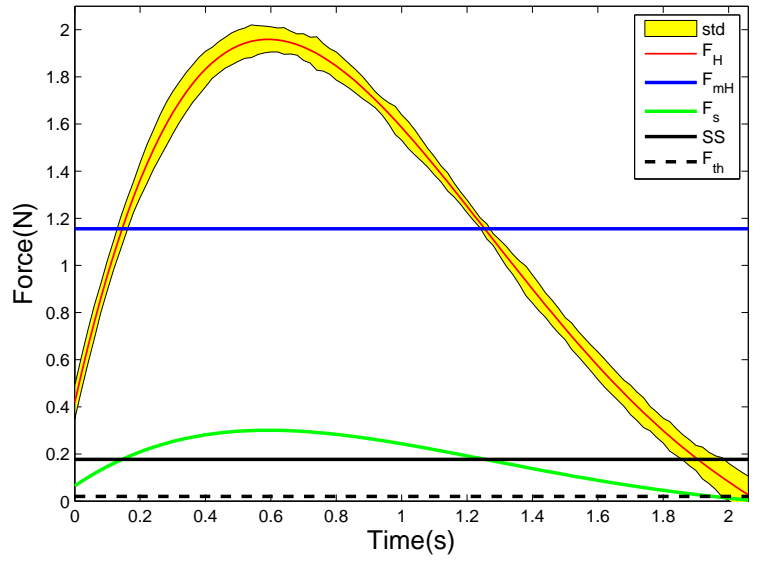

Fig. 7. Impedance parameter adpatation: An example shows how the session specific force profile $\left(F_{\mathrm{S}}\right)$ is constructed from the session score $(S S)$ and the healthy participant's force profile $\left(F_{\mathrm{H}}\right)$. The yellow region is showing the standard deviation of the healthy population mean of the force profile $\left(F_{\mathrm{H}}\right)$ The score $S S$ for this particular session was $0.18 \mathrm{~N}$, which is equal to the average force generated in that session. The average force in case of the healthy participant $\left(F_{\mathrm{mH}}\right)$ was $1.18 \mathrm{~N}$. The threshold force $F_{\text {th }}$ was set to $0.02 \mathrm{~N}$.

$F_{\text {s. }}$ One example is shown in Fig. 7 . It can be seen that the average force applied by a healthy subject was $1.18 \mathrm{~N}$ and this force was lowered to $0.18 \mathrm{~N}$ for the patient to enable him/her to move the fingers with an enhanced velocity, as long as the applied force is above the threshold force level. As the force generation capacity of the patients is initially quite low and sometimes it goes below the threshold force level, they take much more time than a healthy person to complete each $P U$.

\section{E. Participants}

Four chronic hemiparetic stroke (ischemic) patients (3 females, 1 male, age 62.75 \pm 5.56 (range 56-69)) who had partial impairment in finger extension and grasping ability were recruited for the six week long single arm non-randomized clinical trial. The mean time sinnce stroke was $23.25 \pm 3.77$ months (range 19-28 months). All the participants were first time stroke victims. All participants provided written informed consent for their participation and this study was approved by the University Research Ethics Committee of the Ulster University, Northern Ireland, UK. All research procedures were carried out in accordance with approved institutional guidelines and regulations. Inclusion criteria were as follows: ischemic stroke resulting in hand disability, time since stroke onset greater than 6 months, age between 18-80 years (both inclusive), and no history of neurological condition. Exclusion criteria were as follows: severe deficits in cognition (MiniMental State Examination (MMSE [45]) score <21), pregnant or breastfeeding. The trial is registered with the ISRCTN with reference number ISRCTN13139098. The demographics of the patients are shown in Table IV.

The functional recovery of the hand was measured in terms of grip-strength (GS) using a dynamometer, and Action Research Arm Test (ARAT) [46] which are standard measurements of motor outcome [47], [48]. Participants were seated on a chair with their feet touching the ground. The elbow joint was at right angle, while applying gripping force on the dynamometer. Three readings were averaged to obtain each GS measurement. ARAT is a reliable way of testing the upper-limb functionality by checking the grasp, grip, pinch and grossmovement activities. The total score associated with the test is 57, while the minimal clinically important difference (MCID) limit is 5.7 [47]. Five such GS and ARAT measurements were obtained: one at the beginning, one at the end of the entire hand therapy, and three in the intermediate sessions.

The participants had partial disability in their hand and the baseline impairment was measured using the clinical scales of Grip-Strength (GS) and Action Research Arm Test (ARAT), which were $8.71 \pm 4.75 \mathrm{~kg}$ and $23.5 \pm 12.5$ (out of total score of 57) respectively. This disability was due to Ischemic stroke in different parts of the brain as shown in Table IV last column.

\section{Results}

Results describe the change in the force generation capacity of the patient participants. The average fingertip force per session $(S S)$, the change in the average operating velocity of the exoskeleton, and the required level of assistance are also analyzed The variation of the impedance parameters $M, C$ across all the therapy sessions is also obtained for each participant, to check the effectiveness of challenge based strategy in updating the difficulty level of the task.

The performance of participants in the first, middle and the last therapy session is shown in Fig. 8. For each participant, the top row (Fig. 8a-8d) shows the variation of net force $F_{\text {net }}$ during grasping motion in one of the practice units in these three sessions. The time taken for each motion was around 1520 seconds. The corresponding force profile $F_{\mathrm{s}}$, based on the score $S S$ of that session, is shown in the bottom row. Figure 8 shows that the session score $S S$ has increased continuously from the first session till the last one. Details of this increase are shown in Fig 9a. We can also see that the profile of $F_{\mathrm{s}}$ has shifted closer to that of $F_{\mathrm{H}}$, as the session score $S S$ has increased. This enhancement in the force generation capability is observed for all the participants. The $F_{\text {net }}$ profile also gives us the idea of when the system went into passive assistance mode, from the time segments where $F_{\text {net }}$ fell below the threshold force level $\left(F_{\mathrm{th}}=0.02 \mathrm{~N}\right)$. Details of the reduction in the assistance level, as the sessions progressed are given in Fig. 9c.

From Fig. 9a, it can be seen that the highest increment in $S S$ was found to be $0.17 \mathrm{~N}$ in case of the participant P01, while the least increment of $0.12 \mathrm{~N}$ was seen in case of the participant P03. The group mean of the score $S S$ in the first session was $0.04 \mathrm{~N}$, while in the last session, it increased to $0.19 \mathrm{~N}$; an overall increase of $0.15 \mathrm{~N}$. Thus, given the healthy individual's score $S S$ of $1.18 \mathrm{~N}$ (Fig. 7), group mean increase in $S S$ of the participants was from $3.39 \%$ to $16.10 \%$. An overall increase in the average velocity was also seen (Fig. 9b). Each point in Fig. 9b gives an estimate of how fast the participant operated the exoskeleton, while performing the practice units (PU) within a session. The group mean increment in the average velocity is $11 \mathrm{deg} / \mathrm{s}$, with the highest 
TABLE IV

Demographics of the Patient Participants

\begin{tabular}{|c|c|c|c|c|c|c|c|}
\hline $\begin{array}{c}\text { Participant } \\
\text { ID }\end{array}$ & $\begin{array}{c}\text { Age } \\
\text { (years) }\end{array}$ & Sex & $\begin{array}{c}\text { Affected } \\
\text { Hand }\end{array}$ & $\begin{array}{c}\text { MMSE } \\
\text { Score }\end{array}$ & $\begin{array}{c}\text { Time Since Stroke } \\
\text { (months) }\end{array}$ & Stroke Type & Affected Brain location \\
\hline P01 & 61 & F & Right & $30 / 30$ & 22 & Ischemic & Left side of Pons \\
\hline P02 & 56 & M & Left & $30 / 30$ & 28 & Ischemic & $\begin{array}{c}\text { Right middle cerebral } \\
\text { Artery }\end{array}$ \\
\hline P03 & 69 & F & Right & $30 / 30$ & 24 & Ischemic & $\begin{array}{c}\text { Left frontal lobe peri-ventricular } \\
\text { and basal ganglia region }\end{array}$ \\
\hline P04 & 65 & F & Left & $30 / 30$ & 19 & Ischemic & Thalamus \\
\hline Mean (SD) & $62.75(5.56)$ & & & $30(0)$ & $23.25(3.77)$ & & \\
\hline
\end{tabular}

increment of $0.16 \mathrm{deg} / \mathrm{s}$ for the participant P04 and the least increment of $0.03 \mathrm{deg} / \mathrm{s}$ for the participant P01. This shows that, although the impedance (difficulty) level of the system increased when there was an increase in the SS level, all the participants were found motivated by the increased challenge level.

As the participants got more and more engaged in the rehabilitation process and increased their effort, a corresponding reduction in the passive assistance provided by the exoskeleton was seen. This means that they were able to maintain their fingertip force above the threshold level more consistently. The assistance level was calculated as a percentage of time the exoskeleton controller went into passive assistance mode as compared to the user-driven mode. Figure $9 \mathrm{c}$ shows that for all the participants, the assistance level was reduced at the end of the therapy. The maximum reduction in the assistance level was found to be $77.85 \%$ for the participant P01, while the minimum was found to be $19.75 \%$ for the participant P04. The group mean reduction of $37.84 \%$ in the required assistance level was also found to be significant $(p-$ value $<0.05$ ).

Variation of $M$ and $C$ parameters of the impedance model is shown in Figs. 10 and 11, which show that both the $M$ and $C$ parameters increased through the sessions with a group mean change of $0.0027 \mathrm{Ns}^{2} / \mathrm{deg}$ and $0.0021 \mathrm{Ns} / \mathrm{deg}$ in $M$ and $C$ respectively.

A group mean increase of $9.04 \mathrm{~kg}$ is observed over the baseline average GS of $8.71 \mathrm{~kg}$ in the begining of the therapy. The GS measurements are also correlated with the SS (Fig. 12 ), considering the $S S$ values on those days only when the GS measurements were taken. A significantly $(p<0.01)$ high correlation is seen between GS and $S S$ for all participants. From the Fig. 13(a) it can be seen that all the participants were able to increase their ARAT scores throughout the clinical trial, while Fig. 13(b) shows that the group mean had changed (mean \pm std $=+23.75 \pm 2.5)$ significantly $(p$-value $<0.05)$ from the pre $(23.5 \pm 12.5)$ to post $(47.25 \pm 12.12)$ intervention, which is also clinically significant as it is found to be greater than the MCID limit.

\section{Discussion}

Continuous increase in the impedance level (Figs. 10 and 11) is an outcome of the continuous increase in session score of the participants (Fig. 9a), which in turn is due to the increased effort put by them in the subsequent sessions. This shows that the proposed impedance updating strategy was successful in providing the required amount of challenge at each session to motivate the participants towards greater functional recovery of their impaired hand. The enhancement of the average force applied (the session score $S S$ ), the reduction in the required assistance and the increment in the impedance throughout the sessions prove the feasibility and effectiveness of the impedance adaptation based trigged assistance strategy, for updating the challenge level of the poststroke hand therapy.

The current investigation of hand functional recovery using the robotic exoskeleton is significant in many aspects. From the design point of view, a underactuated design helps in making a device fully portable, lightweight, and easily deployable for use in hospital or home. The minimization of the design complexity is based on the idea that grasping needs can be met with lesser number of degrees of freedom (DOF) [49] and it is often considered enough to use one DOF per phalanx for neurorehabilitation [50]. Unlike cable driven mechanisms, direct drive is less complicated as the effects of cable routing, friction and backlash are avoided [51]. Linkages have the advantage of efficient power transmission and bi-directional actuation [52]. The problem of joint axis misalignment was circumvented by using four-bar linkages for path generation. All the mechanisms and drives are placed on the back of the hand which is supported through an arm rest. Thus, little weight is felt on the patient's fingers during the rehabilitation process.

The musclular model free approach of providing assistance to the patients also simplifies the exoskeleton control and the therapeutic process. First of all, it is difficult to construct the patient specific model of human motor system [53], or predefine trajectory for each individual, which sometimes needs the involvement of a human therapist [54]. Therefore, a similar approach of not forcing the patients to follow a predefined joint angle trajectory is chosen, like some other studies [1], [23], [24]. While Crocher et al. [24] used joint synergies instead of predefined trajectories for promoting free interaction, here we used an impedance model, adjusted as per the patient's performance level, without putting any velocity/acceleration constraints.

Continuous measurement of the force contribution is often made indirectly from the robot's torque [40], as it is difficult 

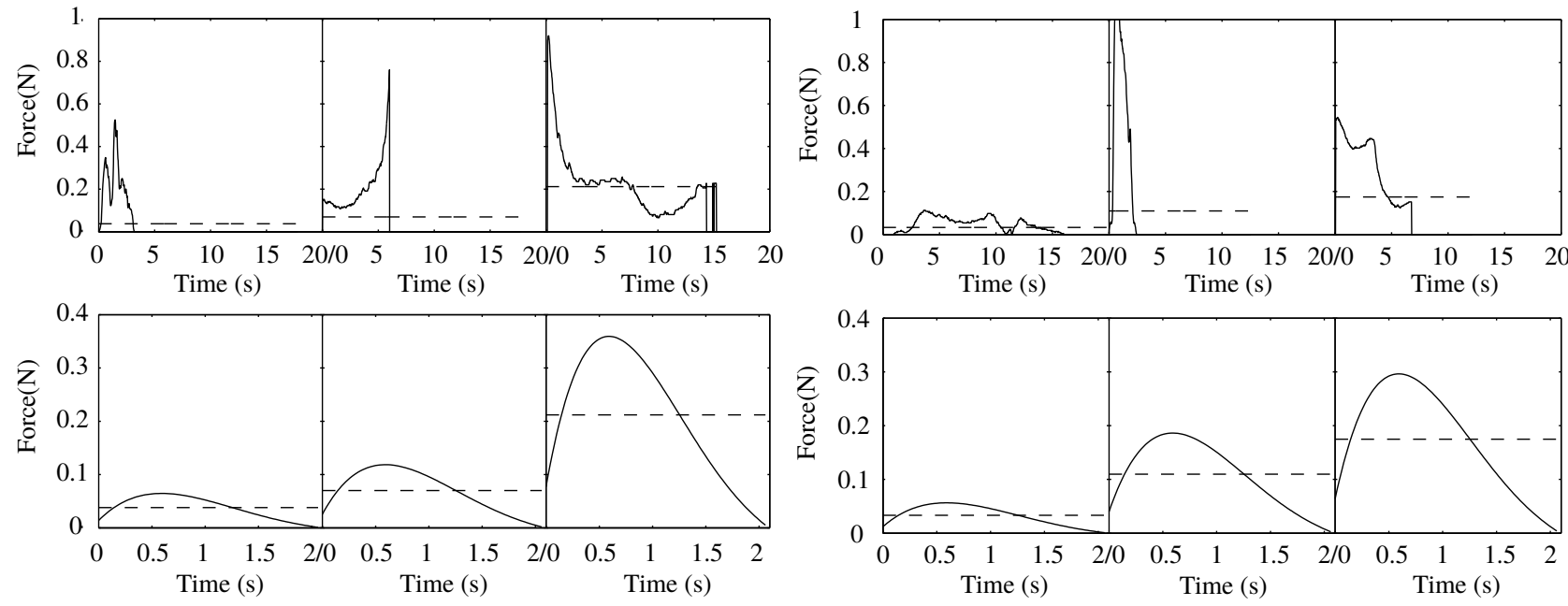

(a)

- force $(\mathrm{t})$

(b)
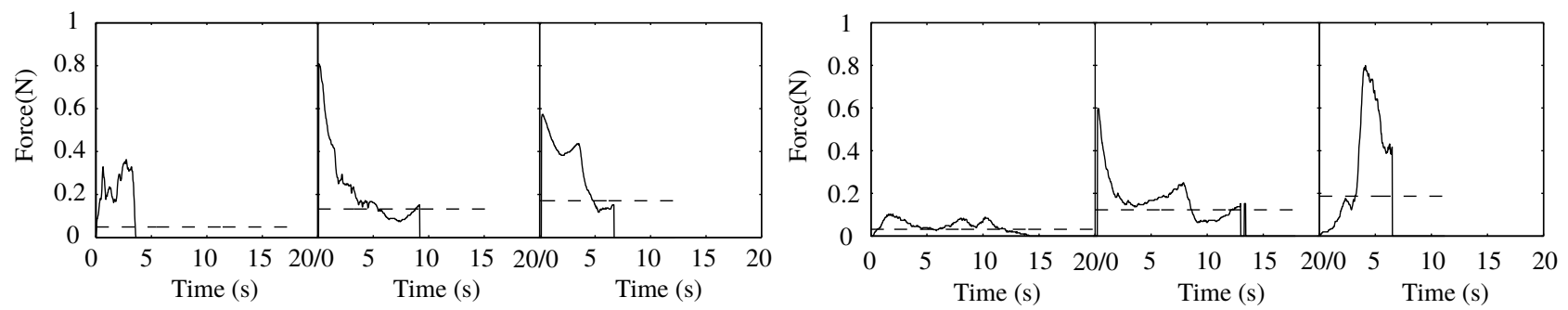

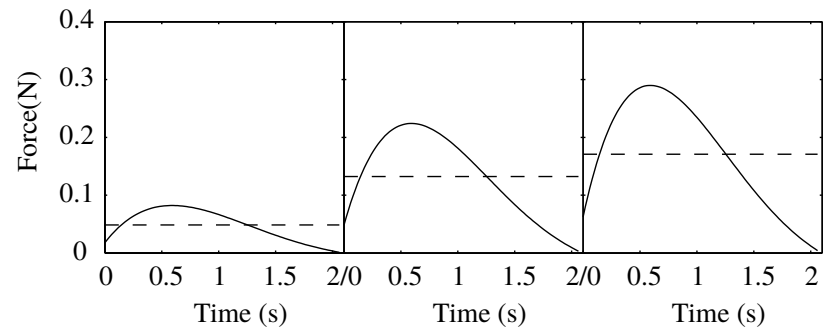

(c)

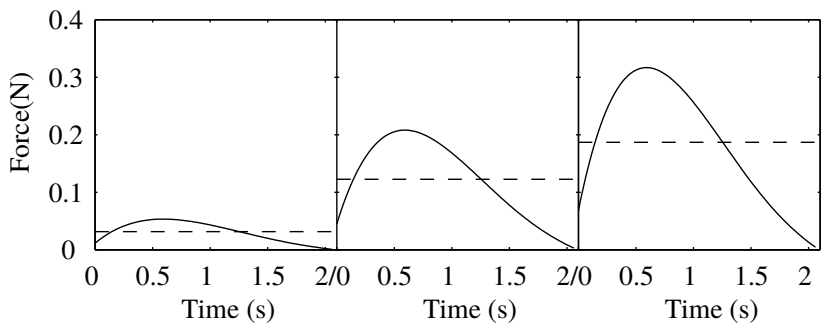

(d)

Fig. 8. Change in the force profiles of the participants (a) P01, (b) P02, (c) P03 and (d) P04: The top row in each subfigure (a, b, c, d) shows the net force $F_{\text {net }}$ during grasping motion in the first, the middle and the last sessions (left to right). The bottom row shows the modified force profile $F_{\mathrm{s}}$ obtained using the method described in Section III-D. The horizontal broken line in each graph shows the average force level.

to mount sensors in the small space of the finger surface [55]. However, the acquisition of clean torque signal is difficult, specially with noisy strain gauges. Hence, we measured the patient's force contribution directly using the FSRs mounted inside the finger-inserts. The fingertip inserts also allow some variation in the finger lengths of different patients. This type of integration of force sensors can also be found in an earlier study [39]. The difficulty level of the task is normally controlled by the human therapist [56]. This feature has been automated by the performance based update of impedance parameters in our system. The force feedback encourages the patient to exert more effort leading towards higher recovery [57]. The switching between the two modes (active nonassist and passive assistance) is decided by the magnitude of the force generated by the patient. This kind of time triggered approaches are popular with exoskeletons such as Gentle/G [58] and Rupert [59]. Thus the inter-modal switching approach with real-time force feedback helps in avoiding the slacking effect, commonly associated with passive assistance.

The results obtained from the study clearly show the increment of fingertip force generation capacity with a reduction in the assistance level and increase in the range of motion. The results conform to the notion that the assistance requirement is reduced with the enhancement of the performance level of the patients [40]. The positive effect of resistance driven exercises on poststroke motor function is also supported by some nonrobotic studies [60], [61]. The present study also reaffirms the validity of robot mediated hand therapy, which has been reported in many other previous studies [62], [63].

Some of the shortcomings of the device include its inability to deal with tremor suppression [39] and higher levels of hypertonia [64], especially in those cases where the muscle tone increase by repetitive movements. Due to these factors we couldn't accommodate such patients in the study. Moreover, as the BCI based mental practice phase was also included following the physical practice phase using the proposed challenge 


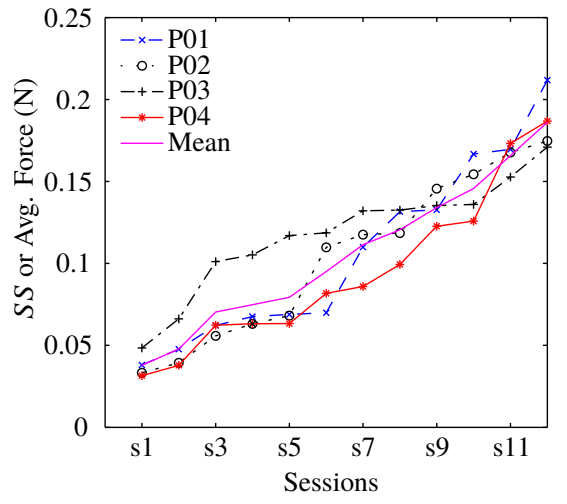

(a)

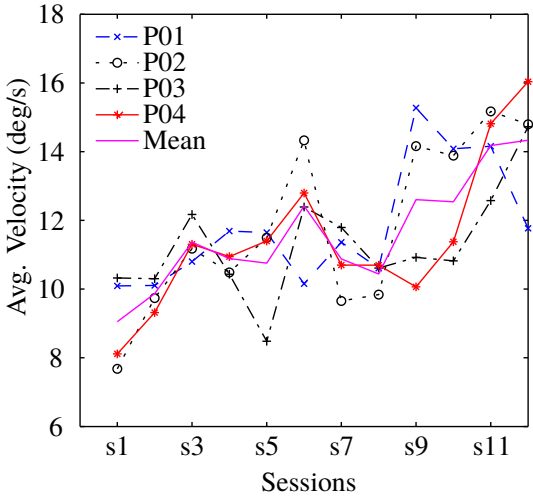

(b)

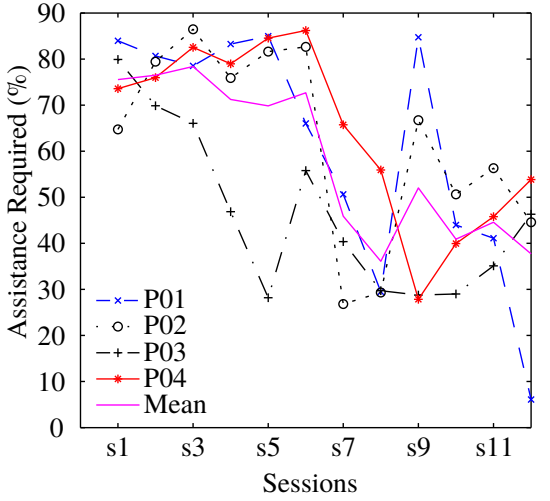

(c)

Fig. 9. The variation in (a) session score $(S S)$, (b) velocity and (c) required assistance level with the therapy sessions.

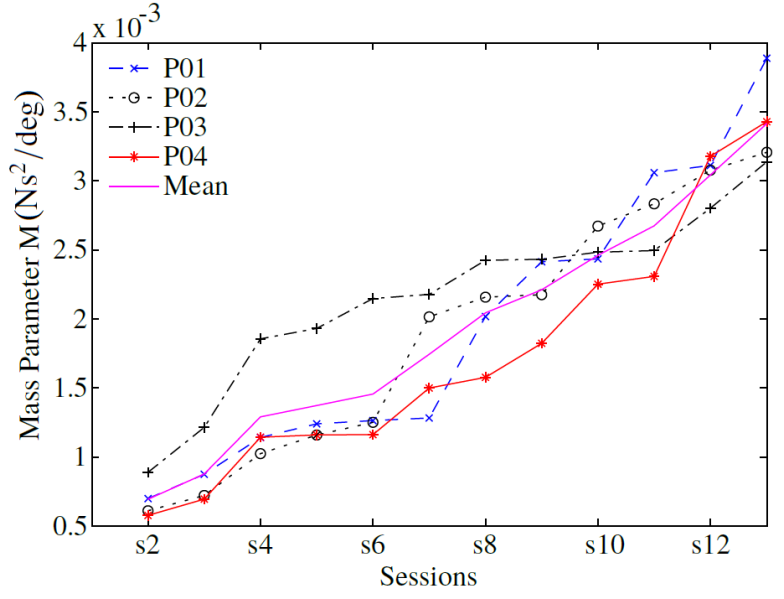

Fig. 10. The mass parameter $M$ for the therapy sessions.

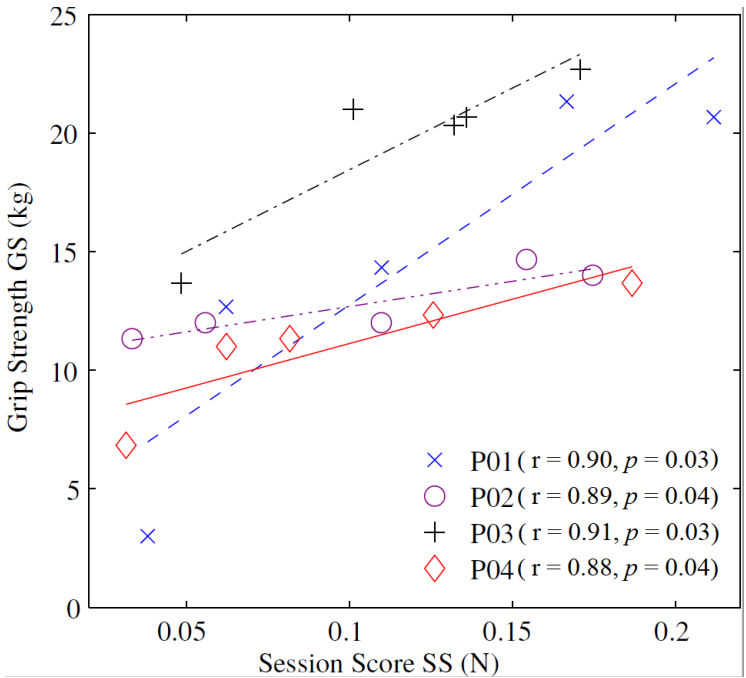

Fig. 12. Variation in the grip-strength (GS) with the session score $(S S)$ for all participants P01-P04: Significantly $(\mathrm{p}<0.01)$ high correlation coefficients (r) between GS and $S S$ are found. The lines show the linear-least-square fit for the data-points corresponding to each participant.
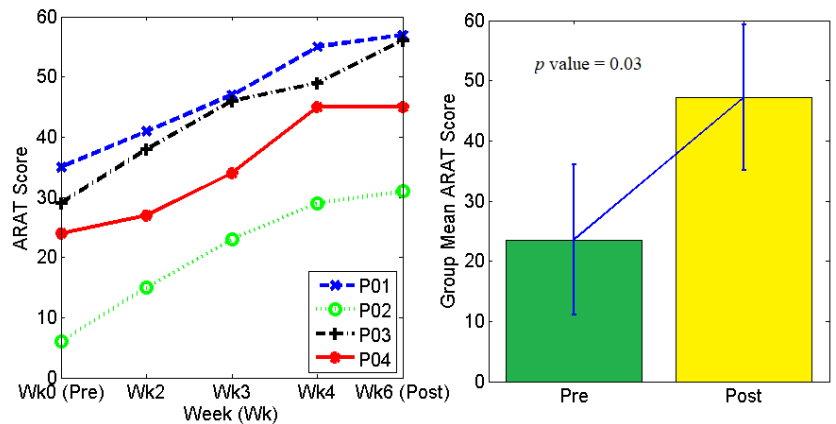

Fig. 13. The variation in ARAT scores for individual participants over the clinical trial period are shown in (a), while the group mean change in the ARAT score from Pre to Post intervention is shown in (b). The change in the group mean of ARAT is found to be both statistically significant $(p$-value $<0.05)$ and greater than the MCID limit of 5.7.

Fig. 11. The damping coefficient $C$ for the therapy sessions. 
based strategy, the functional recovery of the participants may have been affected by both of these phases. Hence, controlled trials need to be performed in future to compare the effect of each phase on the total hand functional recovery. It is worth mentioning that the preliminary results obtained in this study with a small number of patients need to be further validated on large patient cohorts. However, the fingertip force generation capacity, in terms of the session score $(S S)$ is found to be significantly correlated with the offline assessment of the hand functional recovery in terms of the grip-strength (GS) in all the participants. This shows that the fingertip force measurements, via the force sensors, can facilitate direct monitoring of the progress of the motor recovery, without needing the off-line measurement of the grip strength. As the impedance parameters are adapted to adjust the challenge level of the task as per the user performance, the proposed system may also offer personalized exercises for a rehabilitative hand therapy.

\section{Conclusion}

The study clearly demonstrates the feasibility of a novel challenge-based neurorehabilitation strategy using a fully wearable, portable and lightweight hand-exoskeleton, as the patients are able to operate at higher impedance level, requiring lesser assistance as the therapy sessions progress. The novel combination of active non-assist mode along with triggered passive assistance promotes free interaction during the rehabilitation process. The new force generation capacity based performance metric, $S S$ is also found to be significantly correlated with GS, providing a way of online motor recovery assessment using hand-exoskeleton mounted force sensors. Finally, significant positive changes in ARAT shows potential clinical effectiveness of the study. However, controlled trials need to be performed in larger patient population in future to identify the individual contribution of the BCI based mental practice phase and the physical practice phase using the proposed challenge-based strategy on the hand functional recovery.

\section{ACKNOWLEDGMENT}

This work is supported by Department of Science and Technology (DST), India and UK India Education and Research Initiative (UKIERI) Thematic Partnership project: UKIERI-DST2013-14/126, DST/INT/UK/P- 80/2014 and DST-UKIERI2016-17-0128.

\section{REFERENCES}

[1] J.-C. Metzger, O. Lambercy, A. Califfi, D. Dinacci, C. Petrillo, P. Rossi, F. M. Conti, and R. Gassert, "Assessment-driven selection and adaptation of exercise difficulty in robot-assisted therapy: a pilot study with a hand rehabilitation robot," Journal of NeuroEngineering and Rehabilitation, vol. 11, no. 1 , p. 154, Nov 2014.

[2] R. W. Teasell, N. C. Foley, S. K. Bhogal, and M. R. Speechley, "An evidence-based review of stroke rehabilitation," Topics in Stroke Rehabilitation, vol. 10, no. 1, pp. 29-58, 2003, pMID: 12970830.

[3] H. I. Krebs, B. T. Volpe, D. Williams, J. Celestino, S. K. Charles, D. Lynch, and N. Hogan, "Robot-aided neurorehabilitation: A robot for wrist rehabilitation," IEEE Transactions on Neural Systems and Rehabilitation Engineering, vol. 15, no. 3, pp. 327-335, Sept 2007.
[4] F. Wang, D. E. Barkana, and N. Sarkar, "Impact of visual error augmentation when integrated with assist-as-needed training method in robot-assisted rehabilitation," IEEE Transactions on Neural Systems and Rehabilitation Engineering, vol. 18, no. 5, pp. 571-579, Oct 2010.

[5] L. Dipietro, H. I. Krebs, B. T. Volpe, J. Stein, C. Bever, S. T. Mernoff, S. E. Fasoli, and N. Hogan, "Learning, not adaptation, characterizes stroke motor recovery: Evidence from kinematic changes induced by robot-assisted therapy in trained and untrained task in the same workspace," IEEE Transactions on Neural Systems and Rehabilitation Engineering, vol. 20, no. 1, pp. 48-57, Jan 2012.

[6] P. Agarwal, Y. Yun, J. Fox, K. Madden, and A. D. Deshpande, "Design, control, and testing of a thumb exoskeleton with series elastic actuation," The International Journal of Robotics Research, vol. 36, no. 3, pp. 355375, 2017.

[7] M. Cortese, M. Cempini, P. R. de Almeida Ribeiro, S. R. Soekadar, M. C. Carrozza, and N. Vitiello, "A mechatronic system for robotmediated hand telerehabilitation," IEEE/ASME Transactions on Mechatronics, vol. 20, no. 4, pp. 1753-1764, Aug 2015.

[8] J. Sabater-Navarro, N. Garcia, D. Ramos, E. Camayo, and A. Vivas, "Hand neuro-rehabilitation system using nitinol spring actuators," Robotics and Autonomous Systems, vol. 63, pp. 50 - 56, 2015.

[9] S. W. Lee, K. A. Landers, and H. Park, "Development of a biomimetic hand exotendon device (biomhed) for restoration of functional hand movement post-stroke," IEEE Transactions on Neural Systems and Rehabilitation Engineering, vol. 22, no. 4, pp. 886-898, July 2014.

[10] F. Zhang, L. Hua, Y. Fu, H. Chen, and S. Wang, "Design and development of a hand exoskeleton for rehabilitation of hand injuries," Mechanism and Machine Theory, vol. 73, pp. 103 - 116, 2014.

[11] U. Jeong, H.-K. In, and K.-J. Cho, "Implementation of various control algorithms for hand rehabilitation exercise using wearable robotic hand," Intell. Serv. Robot., vol. 6, no. 4, pp. 181-189, Oct. 2013.

[12] N. S. K. Ho, K. Y. Tong, X. L. Hu, K. L. Fung, X. J. Wei, W. Rong, and E. A. Susanto, "An emg-driven exoskeleton hand robotic training device on chronic stroke subjects: Task training system for stroke rehabilitation," in 2011 IEEE International Conference on Rehabilitation Robotics, June 2011, pp. 1-5.

[13] R. C. V. Loureiro, W. S. Harwin, R. Lamperd, and C. Collin, "Evaluation of reach and grasp robot-assisted therapy suggests similar functional recovery patterns on proximal and distal arm segments in sub-acute hemiplegia," IEEE Transactions on Neural Systems and Rehabilitation Engineering, vol. 22, no. 3, pp. 593-602, May 2014.

[14] O. Sandoval-Gonzalez, J. Jacinto-Villegas, I. Herrera-Aguilar, O. Portillo-Rodiguez, P. Tripicchio, M. Hernandez-Ramos, A. FloresCuautle, and C. Avizzano, "Design and development of a hand exoskeleton robot for active and passive rehabilitation," International Journal of Advanced Robotic Systems, vol. 13, no. 2, p. 66, 2016.

[15] S. Ueki, H. Kawasaki, S. Ito, Y. Nishimoto, M. Abe, T. Aoki, Y. Ishigure, T. Ojika, and T. Mouri, "Development of a hand-assist robot with multidegrees-of-freedom for rehabilitation therapy," IEEE/ASME Transactions on Mechatronics, vol. 17, no. 1, pp. 136-146, Feb 2012.

[16] H. Yap, J. Lim, J. Goh, and C. Yeow, "Design of a soft robotic glove for hand rehabilitation of stroke patients with clenched fist deformity using inflatable plastic actuators," J. Med. Devices., vol. 10, no. 4, pp 044 504-044 504-6, Aug 2016.

[17] J. Metzger, O. Lambercy, and R. Gassert, "Performance comparison of interaction control strategies on a hand rehabilitation robot," in 2015 IEEE International Conference on Rehabilitation Robotics (ICORR), Aug 2015, pp. 846-851.

[18] A. Chiri, N. Vitiello, F. Giovacchini, S. Roccella, F. Vecchi, and M. C. Carrozza, "Mechatronic design and characterization of the index finger module of a hand exoskeleton for post-stroke rehabilitation," IEEE/ASME Transactions on Mechatronics, vol. 17, no. 5, pp. 884-894, Oct 2012.

[19] C. L. Jones, F. Wang, R. Morrison, N. Sarkar, and D. G. Kamper, "Design and development of the cable actuated finger exoskeleton for hand rehabilitation following stroke," IEEE/ASME Transactions on Mechatronics, vol. 19, no. 1, pp. 131-140, Feb 2014.

[20] E. B. Brokaw, I. Black, R. J. Holley, and P. S. Lum, "Hand spring operated movement enhancer (handsome): A portable, passive hand exoskeleton for stroke rehabilitation," IEEE Transactions on Neural Systems and Rehabilitation Engineering, vol. 19, no. 4, pp. 391-399, Aug 2011.

[21] C. Pacchierotti, S. Sinclair, M. Solazzi, A. Frisoli, V. Hayward, and D. Prattichizzo, "Wearable haptic systems for the fingertip and the hand: Taxonomy, review, and perspectives," IEEE Transactions on Haptics, vol. PP, no. 99, pp. 1-1, 2017. 
[22] L. Marchal-Crespo and D. J. Reinkensmeyer, "Review of control strategies for robotic movement training after neurologic injury," Journal of NeuroEngineering and Rehabilitation, vol. 6, no. 1, p. 20, Jun 2009.

[23] C. Obayashi, T. Tamei, and T. Shibata, "Assist-as-needed robotic trainer based on reinforcement learning and its application to dart-throwing," Neural Networks, vol. 53, no. Supplement C, pp. 52 - 60, 2014.

[24] V. Crocher, A. Sahbani, J. Robertson, A. Roby-Brami, and G. Morel, "Constraining upper limb synergies of hemiparetic patients using a robotic exoskeleton in the perspective of neuro-rehabilitation," IEEE Transactions on Neural Systems and Rehabilitation Engineering, vol. 20, no. 3, pp. 247-257, May 2012.

[25] R. Colombo, F. Pisano, S. Micera, A. Mazzone, C. Delconte, M. C. Carrozza, P. Dario, and G. Minuco, "Robotic techniques for upper limb evaluation and rehabilitation of stroke patients," IEEE Transactions on Neural Systems and Rehabilitation Engineering, vol. 13, no. 3, pp. 311324, Sept 2005.

[26] C. D. Takahashi, L. Der-Yeghiaian, V. H. Le, and S. C. Cramer, "A robotic device for hand motor therapy after stroke," in 9th International Conference on Rehabilitation Robotics, 2005. ICORR 2005., June 2005, pp. $17-20$.

[27] J. Huang, X. Tu, and J. He, "Design and evaluation of the rupert wearable upper extremity exoskeleton robot for clinical and in-home therapies," IEEE Transactions on Systems, Man, and Cybernetics: Systems, vol. 46, no. 7, pp. 926-935, July 2016.

[28] R. C. V. Loureiro and W. S. Harwin, "Reach amp; grasp therapy: Design and control of a 9-dof robotic neuro-rehabilitation system," in 2007 IEEE 10th International Conference on Rehabilitation Robotics, June 2007, pp. 757-763.

[29] F. Amirabdollahian, R. Loureiro, E. Gradwell, C. Collin, W. Harwin, and G. Johnson, "Multivariate analysis of the fugl-meyer outcome measures assessing the effectiveness of gentle/s robot-mediated stroke therapy," Journal of NeuroEngineering and Rehabilitation, vol. 4, no. 1, p. 4, Feb 2007.

[30] R. Colombo, F. Pisano, S. Micera, A. Mazzone, C. Delconte, M. C. Carrozza, P. Dario, and G. Minuco, "Robotic techniques for upper limb evaluation and rehabilitation of stroke patients," IEEE Transactions on Neural Systems and Rehabilitation Engineering, vol. 13, no. 3, pp. 311324, Sept 2005.

[31] R. Colombo, F. Pisano, S. Micera, A. Mazzone, C. Delconte, M. Carrozza, P. Dario, and G. Minuco, "Assessing mechanisms of recovery during robot-aided neurorehabilitation of the upper limb," Neurorehabilitation and Neural Repair, vol. 22, no. 1, pp. 50-63, 2008, pMID 17626223.

[32] P. Maciejasz, J. Eschweiler, K. Gerlach-Hahn, A. Jansen-Toy, and S. Leonhardt, "A survey on robotic devices for upper limb rehabilitation," Journal of neuroengineering and rehabilitation, vol. 11, p. 3, 012014.

[33] S. S. Nishad, A. Dutta, and A. Saxena, "Design and control of a three finger hand exoskeleton for translation of a slender object," in 2014 11th International Conference on Ubiquitous Robots and Ambient Intelligence (URAI), Nov 2014, pp. 179-184.

[34] M. F. Orlando, H. Akolkar, A. Dutta, A. Saxena, and L. Behera, "Optimal design and control of a hand exoskeleton," in 2010 IEEE Conference on Robotics, Automation and Mechatronics, June 2010, pp. 72-77.

[35] D. Leonardis, M. Barsotti, C. Loconsole, M. Solazzi, M. Troncossi, C. Mazzotti, V. P. Castelli, C. Procopio, G. Lamola, C. Chisari, M. Bergamasco, and A. Frisoli, "An emg-controlled robotic hand exoskeleton for bilateral rehabilitation," IEEE Transactions on Haptics, vol. 8, no. 2, pp. 140-151, April 2015

[36] D. Prattichizzo, F. Chinello, C. Pacchierotti, and M. Malvezzi, "Towards wearability in fingertip haptics: A 3-dof wearable device for cutaneous force feedback," IEEE Transactions on Haptics, vol. 6, no. 4, pp. 506516, Oct 2013.

[37] J. Arata, K. Ohmoto, R. Gassert, O. Lambercy, H. Fujimoto, and I. Wada, "A new hand exoskeleton device for rehabilitation using a three-layered sliding spring mechanism," in 2013 IEEE International Conference on Robotics and Automation, May 2013, pp. 3902-3907.

[38] P. Polygerinos, Z. Wang, K. C. Galloway, R. J. Wood, and C. J. Walsh, "Soft robotic glove for combined assistance and at-home rehabilitation," Robotics and Autonomous Systems, vol. 73, no. Supplement C, pp. 135 - 143, 2015, wearable Robotics.

[39] A. Wege, K. Kondak, and G. Hommel, "Force control strategy for a hand exoskeleton based on sliding mode position control," in 2006 IEEE/RSJ International Conference on Intelligent Robots and Systems, Oct 2006, pp. $4615-4620$.

[40] J. C. Pérez-Ibarra, A. A. G. Siqueira, and H. I. Krebs, "Assist-as-needed ankle rehabilitation based on adaptive impedance control," in 2015
IEEE International Conference on Rehabilitation Robotics (ICORR), Aug 2015, pp. 723-728.

[41] R. Ikeura and H. Inooka, "Variable impedance control of a robot for cooperation with a human," in Proceedings of 1995 IEEE International Conference on Robotics and Automation, vol. 3, May 1995, pp. 30973102 vol.3.

[42] A. Dutta and G. Obinata, "Impedance control of a robotic gripper for cooperation with humans," Control Engineering Practice, vol. 10, pp. 379-389, 042002.

[43] A. Chowdhury, H. Raza, A. Dutta, and G. Prasad, "EEG-EMG based hybrid brain computer interface for triggering hand exoskeleton for neuro-rehabilitation," in Proceedings of the Advances in Robotics, ser. AIR '17. New York, NY, USA: ACM, 2017, pp. 45:1-45:6.

[44] A. Chowdhury, H. Raza, Y. K. Meena, A. Dutta, and G. Prasad, "Online covariate shift detection based adaptive brain-computer interface to trigger hand exoskeleton feedback for neuro-rehabilitation," IEEE Transactions on Cognitive and Developmental Systems, vol. PP, no. 99, pp. 1-1, 2017.

[45] M. F. Folstein, S. E. Folstein, and P. R. McHugh, "âĂIJmini-mental stateâĂİ: A practical method for grading the cognitive state of patients for the clinician," Journal of Psychiatric Research, vol. 12, no. 3, pp. $189-198,1975$.

[46] R. C. Lyle, "A performance test for assessment of upper limb function in physical rehabilitation treatment and research," International Journal of Rehabilitation Research, vol. 4, no. 4, pp. 483-492, 1981.

[47] G. Prasad, P. Herman, D. Coyle, S. McDonough, and J. Crosbie, "Applying a brain-computer interface to support motor imagery practice in people with stroke for upper limb recovery: a feasibility study." Journal of neuroengineering and rehabilitation, vol. 7 , no. 1, p. 60 , 2010.

[48] D. T. Bundy, L. Souders, K. Baranyai, L. Leonard, G. Schalk, R. Coker, D. W. Moran, T. Huskey, and E. C. Leuthardt, "Contralesional brain-computer interface control of a powered exoskeleton for motor recovery in chronic stroke survivors," Stroke, 2017.

[49] P. H Thakur, A. J Bastian, and S. Hsiao, "Multidigit movement synergies of the human hand in an unconstrained haptic exploration task," The Journal of neuroscience, vol. 28, pp. 1271-81, 022008.

[50] J. Sabater-Navarro, N. Garcia, D. Ramos, E. Camayo, and A. Vivas, "Hand neuro-rehabilitation system using nitinol spring actuators," Robotics and Autonomous Systems, vol. 63, no. Part 1, pp. 50 - 56, 2015.

[51] H. Fang, Z. Xie, H. Liu, T. Lan, and J. Xia, "An exoskeleton force feedback master finger distinguishing contact and non-contact mode," in 2009 IEEE/ASME International Conference on Advanced Intelligent Mechatronics, July 2009, pp. 1059-1064.

[52] S. Park, L. Bishop, T. Post, Y. Xiao, J. Stein, and M. Ciocarlie, "On the feasibility of wearable exotendon networks for whole-hand movement patterns in stroke patients," in 2016 IEEE International Conference on Robotics and Automation (ICRA), May 2016, pp. 3729-3735.

[53] L. Marchal-Crespo and D. J Reinkensmeyer, "Haptic guidance can enhance motor learning of a steering task," Journal of motor behavior, vol. 40, pp. 545-56, 122008.

[54] M. T. M. Shahbazi, S. F. Atashzar and R. V. Patel, "Therapist-in-the-loop robotics-assisted mirror rehabilitation therapyâẤr: An assist-as-needed framework," in 2015 IEEE International Conference on Robotics and Automation (ICRA), 2015, pp. 5910-5915.

[55] N. Popescu, D. Popescu, M. Ivanescu, D. Popescu, C. Vladu, and I. Vladu, "Force observer-based control for a rehabilitation hand exoskeleton system," in 2013 9th Asian Control Conference (ASCC), June 2013, pp. 1-6.

[56] J. H. Carr, Movement Science: Foundations for Physical Therapy in Rehabilitation. Aspen Publishers, 2000.

[57] M. A. Perez, B. K. S. Lungholt, K. Nyborg, and J. B. Nielsen, "Motor skill training induces changes in the excitability of the leg cortical area in healthy humans," Experimental Brain Research, vol. 159, no. 2, pp. 197-205, Nov 2004.

[58] R. C. V. Loureiro and W. S. Harwin, "Reach and grasp therapy: Design and control of a 9-dof robotic neuro-rehabilitation system," in 2007 IEEE 10th International Conference on Rehabilitation Robotics, June 2007, pp. 757-763.

[59] J. He, E. J. Koeneman, R. S. Schultz, D. E. Herring, J. Wanberg, H. Huang, T. Sugar, R. Herman, and J. B. Koeneman, "Rupert: a device for robotic upper extremity repetitive therapy," in 2005 IEEE Engineering in Medicine and Biology 27th Annual Conference, 2005, pp. 6844-6847. 
[60] M. M. Ouellette et al., "High-intensity resistance training improves muscle strength, self-reported function, and disability in long-term stroke survivors," Stroke, vol. 35, no. 6, pp. 1404-1409, 2004.

[61] S. L. Morris, K. J. Dodd, and M. E. Morris, "Outcomes of progressive resistance strength training following stroke: a systematic review," Clinical Rehabilitation, vol. 18, no. 1, pp. 27-39, 2004, pMID: 14763717.

[62] C. D. Takahashi, L. Der-Yeghiaian, V. Le, R. R. Motiwala, and S. C. Cramer, "Robot-based hand motor therapy after stroke," Brain, vol. 131, no. 2 , pp. 425-437, 2008.

[63] C. H. Hwang, J. W. Seong, and D.-S. Son, "Individual finger synchronized robot-assisted hand rehabilitation in subacute to chronic stroke: a prospective randomized clinical trial of efficacy," Clinical Rehabilitation, vol. 26, no. 8, pp. 696-704, 2012, pMID: 22261813.

[64] R. Sasha Blue Godfrey, "Clinical effects of using hexorr (hand exoskeleton rehabilitation robot) for movement therapy in stroke rehabilitation," American Journal of Physical Medicine and Rehabilitation, vol. 92, no. 11, pp. 947-958, 2012.

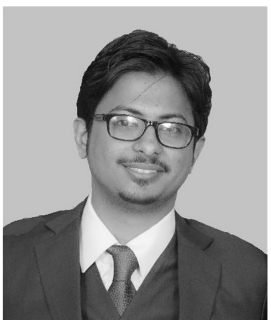

Anirban Chowdhury (S'16) received the B. Tech degree in Electronics and Communication Engineering from Kalyani Govt. Engineering College, Kalyani, India in 2010, the M. Tech degree in Mechatronics and Robotics in 2013 from Bengal Engineering and Science University, Shibpur, Howrah, India. He is in the final stage of his Ph.D. degree in Mechatronics at Indian Institute of Technology Kanpur, Kanpur, India. He was also a visiting researcher at the Intelligent Systems Research Laboratory at the Ulster University, U.K. His research interests are in Brain-Computer Interfaces, Biomedical Signal Processing, Robotics, Artificial Intelligence, and Neurorehabilitation.

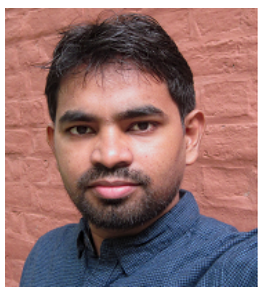

Shyam Sunder Nishad obtained his B. Tech. (2008) and M. Tech. (2013) degrees in Mechanical Engineering from Indian Institute of Technology (IIT) Kanpur. Thereafter, he worked as Project Engineer (2014-16) at IIT Kanpur, in a DST-UKEIRI funded research project on BCI based hand exoskeletons for rehabilitation. Later, he switched over to design and control of insect-like flapping-wing micro-air vehicles during his ongoing $\mathrm{PhD}$ research work at IIT Kanpur. His long term interests lie in design and control of autonomous machines which would find use in almost every aspect of daily life. He was awarded Institute Proficiency medal and Tata Consultancy Services award for best B. Tech. project of the year. He is currently a Visvesvaraya doctoral fellow (2017-) at IIT Kanpur.

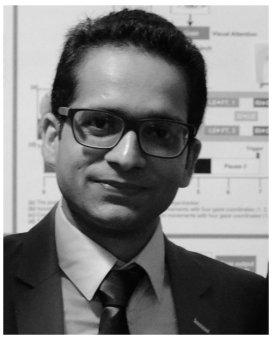

Yogesh Kumar Meena (S'12-M'18) received the B.Tech. and M.Tech. degree in Information Technology from the Indian Institute of Information Technology and Management (IIITM) Gwalior, India in 2010 and the Ph.D. degree in Computer Science from Ulster University, Londonderry, UK in 2018 $\mathrm{He}$ is currently a visiting Post-Graduate Research Scholar in the Faculty of Computing and Intelligent Systems at University of Ulster, UK. Previously, he has worked as a Research Assistant at the School of Computing, Engineering \& Intelligent Systems, Ulster University, UK (May 2017-May 2018) and as an Assistant Professor in the Computer Science \& Information Technology Department at the Sharda Group of Institution, India (July 2010- February 2014). His research interests include human-computer interaction, eye-tracking, brain-computer interface, assistive technology, robotics-based neuro-rehabilitation, machine learning, and bio-physiological signal processing.

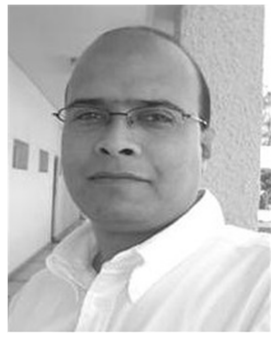

Ashish Dutta (M'07) received the Ph.D. degree in Systems Engineering from Akita University, Akita, Japan. From 1994 to 2000, he was with the Bhabha Atomic Research Center, Mumbai, India, where he researched on telemanipulator design and control for nuclear applications. Since 2002, he has been with the Department of Mechanical Engineering, Indian Institute of Technology Kanpur, Kanpur, India. He was also a Visiting Professor with Nagoya University, Nagoya, Japan, in 2006 and is currently a Visiting Professor with the Kyushu Institute of Technology, Kitakyushu, Japan. His current research interests include biorobotics, robot-human interaction, intelligent control systems, and rehabilitation engineering.

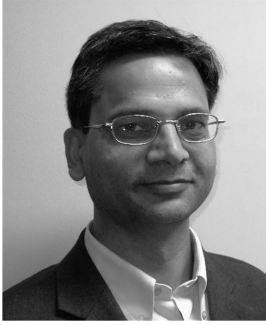

Girijesh Prasad (M'98-SM'07) received the B. Tech degree in Electrical Engineering in 1987, the M. Tech degree in Computer Science and Technology in 1992, and the PhD degree in Electrical Engineering from Queen's University, Belfast, UK in 1997. He currently holds the post of Professor of Intelligent Systems in the School of Computing, Engineering and Intelligent Systems, Ulster University, Magee campus. As an executive member of Intelligent Systems Research Centre at Magee campus, he leads the Neural Systems and Neurotechnology team. He is the Director of Northern Ireland Functional Brain Mapping facility for MEG studies. His research interests are in computational intelligence, brain modelling, brain-computer interfaces and neuro-rehabilitation, and assistive robotic systems. He has published over 230 research papers in international journals, books, and conference proceedings.

Prof. Prasad is a Chartered Engineer and a fellow of the IET and International Academy of Physical Sciences. He is a founder member of IEEE SMC TCs on Brain-Machine Interface Systems. 